\title{
AN AUTOMATIC AND MODULAR STEREO PIPELINE FOR PUSHBROOM IMAGES
}

\author{
Carlo de Franchis ${ }^{\star}$, Enric Meinhardt-Llopis ${ }^{\star}$, Julien Michel ${ }^{\dagger}$, Jean-Michel Morel ${ }^{\star}$ and Gabriele Facciolo ${ }^{\star}$ \\ * CMLA, Ecole Normale Supérieure de Cachan, France - carlo.de-franchis@ens-cachan.fr \\ ${ }^{\dagger}$ CNES - DCT/SI/AP, France
}

\author{
Commission III, WG III/3
}

KEY WORDS: 3D reconstruction, stereo, digital elevation models, remote sensing, pushbroom geometry

\begin{abstract}
:
The increasing availability of high resolution stereo images from Earth observation satellites has boosted the development of tools for producing 3D elevation models. The objective of these tools is to produce digital elevation models of very large areas with minimal human intervention. The development of these tools has been shaped by the constraints of the remote sensing acquisition, for example, using ad hoc stereo matching tools to deal with the pushbroom image geometry. However, this specialization has also created a gap with respect to the fields of computer vision and image processing, where these constraints are usually factored out. In this work we propose a fully automatic and modular stereo pipeline to produce digital elevation models from satellite images. The aim of this new pipeline, called Satellite Stereo Pipeline and abbreviated as $s 2 p$, is to use (and test) off-the-shelf computer vision tools while abstracting from the complexity associated to satellite imaging. To this aim, images are cut in small tiles for which we proved that the pushbroom geometry is very accurately approximated by the pinhole model. These tiles are then processed with standard stereo image rectification and stereo matching tools. The specifics of satellite imaging such as pointing accuracy refinement, estimation of the initial elevation from SRTM data, and geodetic coordinate systems are handled transparently by s2p. We demonstrate the robustness of our approach on a large database of satellite images and by providing an online demo of $\mathrm{s} 2 \mathrm{p}$.
\end{abstract}

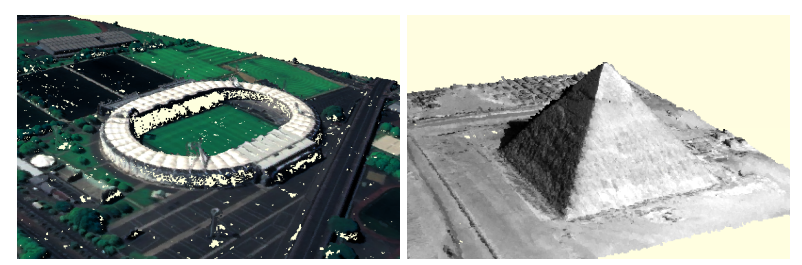

Figure 1: 3D point clouds automatically generated from Pléiades stereo datasets, without any manual intervention, with the $s 2 p$ stereo pipeline. Its implementation can be tested online through a web browser.

\section{INTRODUCTION}

This paper presents an automatic 3D reconstruction pipeline for satellite images, meant to be modular and generic. This work is motivated by the recent availability of high resolution images from new satellites with stereo capabilities such as Pléiades. Even if most of the experiments described here were carried on Pléiades images, our work also applies to images from other satellites such as WorldView, Quickbird, Spot and Ikonos.

The Pléiades constellation is composed of two Earth observation satellites able to deliver images with a resolution of $70 \mathrm{~cm}$ and a swath width of $20 \mathrm{~km}$. Their unique agility allows to capture multiple views of the same target in a single pass. This permits the nearly simultaneous acquisition of two or three images for stereo reconstruction with a small base to height ratio, ranging from 0.15 to 0.8 . Pléiades, as many other Earth observation satellites, acquires images with a pushbroom sensor, which captures them line by line as the satellite moves. The calibration information describing the camera system is provided for all Pléiades images under the form of RPC functions. RPC stands for Rational Polynomial Camera model. Details about these functions are given in appendix A.
The philosophy of the $\mathrm{s} 2 \mathrm{p}$ pipeline is to isolate the $3 \mathrm{D}$ reconstruction problem from the complexities associated to satellite imaging. To that aim the satellite images are processed by small tiles. This permits to locally approximate the pushbroom geometry with a pinhole model, which in turn allows to stereo-rectify the tiles using standard computer vision tools (Hartley and Zisserman, 2004). The rectification error obtained on the tiles is below the tenth of pixel (de Franchis et al., 2014c), improving the state of the art by one order of magnitude (Oh et al., 2010). Each rectified tile is then processed using off-the-shelf stereo matching algorithms.

The pipeline deals transparently with inaccuracies of the sensor attitude (Hanley et al., 2002, Grodecki and Dial, 2003, Fraser and Hanley, 2005), by estimating relative corrections for each tile without needing ground control points (de Franchis et al., 2014b). These local corrections are then combined in a global correction for the entire image, which is used to perform a consistent 3D triangulation. The SRTM information (Shuttle Radar Topography Mission, see section 3.3) is automatically incorporated to identify corresponding regions in both images.

The $s 2 p$ pipeline also handles three-view stereo datasets. In this case two stereo pairs are processed independently, then the resulting elevation models are merged to increase the coverage (see figure 12 for an example). This fully automatic pipeline is available online for testing (de Franchis et al., 2014a).

\subsection{Related works}

Similarly to previous works (Wohlfeil et al., 2012, d'Angelo and Reinartz, 2012, d'Angelo and Kuschk, 2012, Kuschk, 2013), the $s 2 p$ pipeline is fully automated. All tasks that used to be performed manually such as disparity range estimation, tie points selection for RPC refinement, and water masking, are performed automatically thanks to the proper use of SRTM data (Farr et al., 2007) and feature detectors such SIFT (Lowe, 2004). But unlike 
these works, s2p does not include a particular stereo matching algorithm. Instead the main contribution of our work is a complete framework to evaluate any stereo matching algorithm (that works with stereo-rectified images) on satellite pushbroom images.

In the next section we give an overview of the whole pipeline, and in sections 3 to 5 we detail each of its blocks. In section 6 we validate our approach with extensive experimentation carried out using images from Pléiades and WorldView-1.

\section{S2P OVERVIEW}

The s2p pipeline deals with pairs or triplets of images. Pairs and triplets are the standard stereo products proposed by the main commercial providers of satellite images such as DigitalGlobe and Airbus Defense and Space (formerly Astrium).

In case of a stereo triplet, each pair out of the six possible pairs is processed independently, and the resulting $3 \mathrm{D}$ point clouds are then merged. The merging procedure is not discussed in this paper. Figure 2 gives an overview of the processing pipeline for a stereo pair of images. The input images are cut in small tiles, to allow a very precise stereo image rectification. The optimal size of the tiles is discussed in section 3. Then for each tile the calibration data is refined (section 4) and the images are stereorectified (section 3). Each stereo-rectified tile pair is matched using some standard stereo matching algorithm (section 5). The local refinements from all the processed tiles are combined to compute a global correction of the calibration. The triangulation uses the globally corrected calibration data, which is the same for all tiles. This ensures a perfect continuity between the 3D points computed from different tiles.

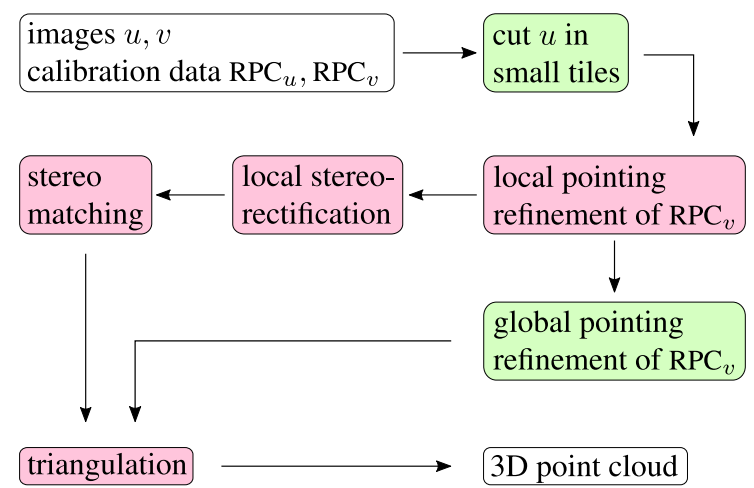

Figure 2: $s 2 p$ overview. The input is a pair of images with their respective rational polynomial camera models, and the output is a digital elevation model given as a georeferenced 3D point cloud. Green blocks are applied to the whole images, while pink blocks are applied on small independent tiles. They can be processed in parallel.

\section{LOCAL STEREO-RECTIFICATION OF PUSHBROOM IMAGES}

Stereo image rectification is a common technique used in 3D reconstruction algorithms. It permits to simplify the search of corresponding points between the images of a stereo pair. However, only images taken with a pinhole camera can be rectified. Pushbroom cameras produce images that are not rectifiable. In this section we study to what extent it is possible to stereo-rectify pushbroom images anyway, in order to use standard matching algorithms from the image processing and computer vision communities for processing satellite stereo pairs. The approach presented here considers the rectification as an auxiliary step for the

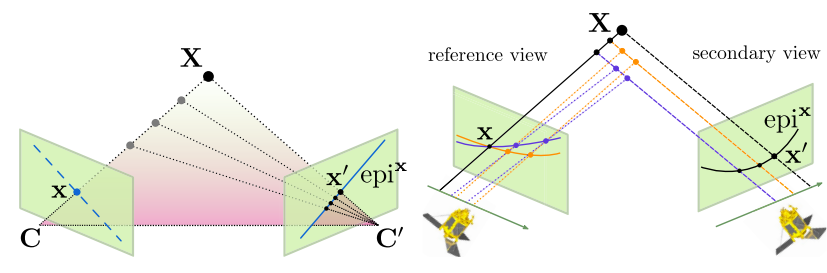

Figure 3: In the pinhole case the epipolar plane defines a correspondence between epipolar lines. In the pushbroom case the projection of a 3-space ray on the secondary view generates a ruled quadric. The projection of this quadric on the reference view contains many epipolar curves: epipolar curves are not conjugate.

computation of stereo correspondences, not as a final product. Images are thus processed in small tiles by locally approximating the pushbroom camera with an affine camera model. The explicit modeling of the approximation allows to quantify and control the rectification errors without needing ground control points. Experiments on Pléiades and WorldView-1 images of many kinds of scenes (urban, mountainous, flat) demonstrate that rectification errors can be reduced to one tenth of pixel.

\subsection{The stereo image rectification problem}

Stereo image rectification permits to restrict the search for corresponding image points from the entire image plane to a single line. For any point $\mathbf{x}$ in a view of the pair, the corresponding point $\mathbf{x}^{\prime}$ in the other view, if it exists, lies on the epipolar line of $\mathbf{x}$ denoted by epi ${ }^{\mathbf{x}}$. Conversely $\mathbf{x}$ lies on epi ${ }^{\mathbf{x}^{\prime}}$. The rectification aims to resample the images in such a way that corresponding points are located on the same row, thus simplifying the matching task and permitting to use all classic stereo matching algorithms.

For images taken with pinhole cameras there is a correspondence between the epipolar lines of the two views. All the points $\mathbf{x}^{\prime}$ of the second view lying on the epipolar line epi ${ }^{\mathrm{x}}$ share the same epipolar line in the first view. Epipolar lines epi ${ }^{\mathbf{x}}$ and epi ${ }^{{ }^{\prime}}$ are said to be conjugate. Figure 3 illustrates the conjugacy of epipolar lines. It is well-known (Hartley and Zisserman, 2004) that images can be resampled in order to produce a rectified pair in which the epipolar lines are horizontal and match up between views. Matching rectified images is much simpler than matching the original images, because the search of correspondences is performed along horizontal lines only (Ohta and Kanade, 1985).

Satellite images however can't be rectified because they are taken with pushbroom sensors, for which the pinhole model is invalid. Using various pushbroom camera models (Orun and Natarajan, 1994, Hartley and Gupta, 1997), it can be shown that pushbroom image pairs have non-straight epipolar curves and that these curves are not conjugate, making rectification impossible (Kim, 2000, Habib et al., 2005).

Many solutions have been proposed to circumvent the non rectifiability of pushbroom images. We may group them into three categories:

1. No rectification (Lee et al., 2003, Hirschmüller et al., 2005, Hirschmüller, 2008): many authors propose to keep the original images unchanged and to perform stereo matching by following the non-straight epipolar curves. This approach eliminates the need for stereo image rectification while keeping the benefits of one-dimensional exploration. 
However, non-straight epipolar curves may prevent from applying stereo matching optimizations and from using offthe-shelf correlators.

2. Affine camera approximation (Ono, 1999, Fraser et al., 2004, Morgan et al., 2006, Wang et al., 2011): other authors propose to approximate the pushbroom sensor with an affine camera model. This approach often uses Ground Control Points (GCP) to estimate the affine model for each image, and the overall achieved precision is on the order of one pixel on images from Spot and Ikonos satellites.

3. Polynomial epipolar resampling (Oh et al., 2010, Christophe et al., 2008): Oh et al. show that even if pairs of epipolar curves don't exist in the pushbroom case, for small altitude ranges of the scene one may assume with small error that curve pairs exist. Thus they build whole epipolar curve pairs on Ikonos stereo images by putting together small pieces of corresponding curves. Then they resample the images to transform these curves into straight horizontal lines. They report a maximal error of one pixel. Since their resampling procedure is non-linear, it can't guarantee that straight lines are preserved.

It is important to note that errors in the rectification are critical as they may result in a vertical disparity between corresponding points in the rectified images, which may hurt the performance of the stereo matching. We refer to this vertical disparity as epipolar error. The epipolar error is the ultimate performance measure for the different methods. Current state of the art methods attain errors on the order of one pixel. The method proposed in this section lowers this error by one order of magnitude.

\subsection{In defense of the affine approximation}

A large-scale stereo-rectified pair is not needed for applying a stereo matching algorithm. Thus we propose, like Morgan et al. (Morgan et al., 2006), to approximate the sensor by an affine camera model. But, unlike Morgan, our approximation is made only on small image tiles. This limits the discrepancy between epipolar curves (Oh et al., 2010). It leads in practice to an almost perfect rectification, with a very small epipolar error.

For each locally rectified tile a standard off-the-shelf stereo algorithm can be applied to estimate a horizontal disparity map, with high chances of success thanks to the high precision of the stereo image rectification. The computed correspondences are then transferred back to the coordinate system of the original images. This eliminates the need for stereo-rectifying the full images all at once.

While Morgan et al. use GCPs to estimate the affine camera models, we use the standard computer vision approach for stereo image rectification (Hartley and Zisserman, 2004): first estimate the affine fundamental matrix between the two views, then compute a pair of affine transformations to rectify the images. The fundamental matrix estimation requires only image matches, eliminating the need for GCPs and manual intervention.

The suitability of the affine camera model in approximating a satellite pushbroom sensor can be attributed to Okamoto et al. (Okamoto et al., 1993). Their main arguments are all applicable to Pléiades and WorldView images:

- Altitude differences in the photographed terrain are small in comparison with the flying altitude of the satellite, whose mean is $694 \mathrm{~km}$ for Pléiades.
- The angular field of view of the sensor is narrow. For a full Pléiades image it is less than $2^{\circ}$, and it is much less if one considers only a small tile.

- The acquisition time of such a tile is less than one second, thus the sensor may be assumed to have the same attitude and speed while capturing the scene.

Our locally affine rectification is presented in Algorithm 1, and its main steps are explained in the next subsections. More details and quantitative experiments about this procedure can be found in (de Franchis et al., 2014c).

\subsection{SRTM data}

The Shuttle Radar Topography Mission (SRTM) is an international research effort (Farr et al., 2007) that obtained digital elevation models on a near-global scale at a resolution of three arcseconds, i.e. $90 \mathrm{~m}$. The SRTM data is used, together with the RPC functions, to estimate the altitude range $\left[h_{m}, h_{M}\right]$ of the 3space points imaged in a given tile. This estimation is needed for the computation of the virtual matches used for stereo image rectification.

\subsection{Virtual correspondences generation}

A natural way to compute correspondences between two views is to extract feature points, compute descriptors and match them, as done by SIFT (Lowe, 2004). But this may lead to a set of keypoints all lying on the same plane, i.e. on the ground. This configuration is degenerate and F cannot be computed from it. Even if the keypoints do not exactly lie on the same plane, as relief reduces to zero, the covariance of the estimated F increases (Hartley and Zisserman, 2004). A safer way to estimate F is to use the calibration data (Oh et al., 2010, Tao and $\mathrm{Hu}, 2001$ ) to generate virtual correspondences between the two views.

Given a region $\Omega$ in the reference image and an estimated altitude range $\left[h_{m}, h_{M}\right]$ for the associated 3 -space points (i.e. points that were imaged into $\Omega$ ) $\Omega$ is back-projected on the Earth surface thanks to RPC ${ }^{-1}$. Let denote by $\Gamma=\operatorname{RPC}^{-1}\left(\Omega \times\left[h_{m}, h_{M}\right]\right) \subset$ $\mathbf{R}^{3}$ the back-projected domain, and by $\left(\mathbf{X}_{i}\right)_{i=1, \ldots, N}$ a regular sampling of $\Gamma$. Each 3 -space point $\mathbf{X}_{i}$ is projected on the two images using the associated RPCs, leading to a virtual correspondence $\left(\mathbf{x}_{i}, \mathbf{x}_{i}^{\prime}\right)$. The images contents at locations $\mathbf{x}_{i}$ and $\mathbf{x}_{i}^{\prime}$ may not correspond, but $\mathbf{x}_{i}^{\prime}$ is located on the epipolar curve of $\mathbf{x}_{i}$, and that is enough to estimate a fundamental matrix.

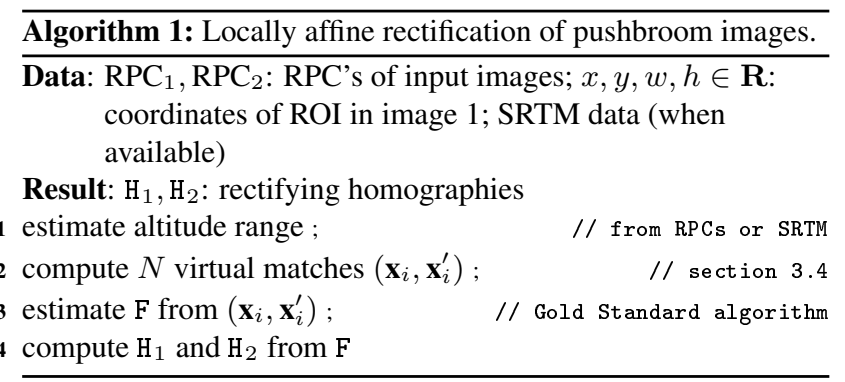

Extensive experiments, presented in section 6 , were carried out on numerous Pléiades datasets. They show that with a tile size of $1000 \times 1000$ pixels the epipolar error is always less than 0.05 pixels. This precision fits all the stereo matching algorithms, thus our pipeline uses a tile size fixed to $1000 \times 1000$ pixels for all Pléiades images. In case of satellites with a different behaviour, the epipolar error can be computed as a preliminary step, and the optimal tile size is automatically selected accordingly. 
It is important to note that this approximation is limited to satellite images. Aerial pushboroom images such as from Leica's ADS 40 or 80 cannot be rectified in that way since a plane cannot fly in a straight line like a satellite.

\section{POINTING CORRECTION}

There is a noticeable bias of a few pixels in the RPC functions (Fraser and Hanley, 2005, Hanley et al., 2002, Grodecki and Dial, 2003). This is inevitable due to the limited precision of the camera calibration. For many purposes, this bias can be ignored, since it typically results in a global offset of the results. However, for stereo matching, the epipolar constraints derived from the parameters of the cameras have to be as precise as possible. The local stereo image rectification algorithm proposed in section 3 relies entirely on the RPC functions. Thus the relative bias between the RPC functions of the images of a stereo pair must be corrected before applying rectification. In this section we propose a method to correct this bias relative to a given reference image. Our method does not rely on ground control points, but on the relative consistency of the image contents; thus, it can be implemented as an automatic pre-processing of the input images.

The knowledge of the projection function RPC and the associated inverse $\mathrm{RPC}^{-1}$ for two images $u$ and $v$ allows to define epipolar curves. If $\mathbf{x}$ is a point in image $u$, then the function

$$
\operatorname{epi}_{u v}^{\mathbf{x}}: h \longmapsto \operatorname{RPC}_{v}\left(\operatorname{RPC}_{u}^{-1}(\mathbf{x}, h)\right)
$$

defines a parametrized curve in the domain of image $v$ containing all the possible correspondences of $\mathbf{x}$ for different altitudes $h$. This curve is called the epipolar curve of the point $\mathbf{x}$. In practice, we observe that these curves are straight line segments which are almost parallel (see figure 4).
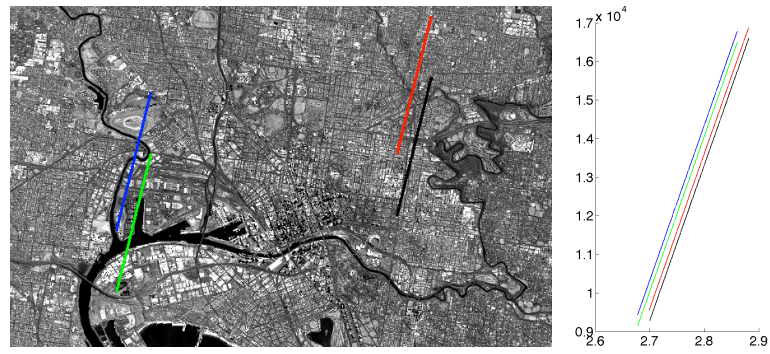

Figure 4: The RPC functions allow to draw the epipolar curves for a pair of images $u$ and $v$ (approx. $16000 \times 40000$ pixels). The left image shows four epipolar curves plotted in the domain of image $v$, they correspond to four points located near the edges of the image $u$. The range of altitudes considered is $h \in[-200,3000]$ meters. The right image shows the same epipolar curves placed closer to facilitate the comparison.

The epipolar curves are used to compute the altitudes of 3-space points which are visible in two images. Suppose that $\mathbf{x}$ is the projection of a point in image $u$, and $\mathbf{x}^{\prime}$ is the projection of the same point in image $v$. Then the epipolar curve of $\mathbf{x}$ passes through $\mathbf{x}^{\prime}$ and the value $h$ for which $\mathbf{x}^{\prime}=\operatorname{epi}_{u v}^{\mathbf{x}}(h)$ is the altitude of the 3space point. An algorithm to compute $h$ is presented in section 5 .

\subsection{The relative pointing error}

Given a pair of corresponding points $\mathbf{x}$ and $\mathbf{x}^{\prime}$ in two images, the epipolar curve of $\mathbf{x}$ may not pass through the point $\mathbf{x}^{\prime}$ (see figure 5). We call this error the relative pointing error. It is not negligible at all, being often of the order of a few pixels.
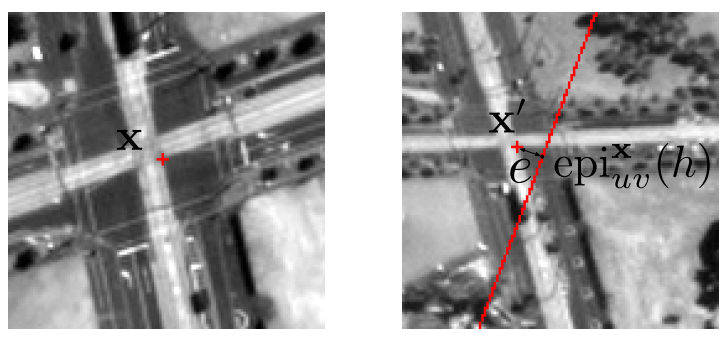

Figure 5: This pair of views of a road intersection highlights the effect of the satellite relative pointing error. Two corresponding points $\mathbf{x}$ and $\mathbf{x}^{\prime}$ are shown, and the epipolar curve of point $\mathbf{x}$ as traced by the RPC doesn't pass through the corresponding point $\mathbf{x}^{\prime}$. The relative pointing error, denoted by $e$, is the distance from the point to the epipolar curve. The altitude of the 3-space point corresponding to $\mathbf{x}$ and $\mathbf{x}^{\prime}$ is approximated by the parameter $h$ for which the epipolar curve passes through the projection of $\mathbf{x}^{\prime}$.

Given two images $u, v$ and a set of correspondences $\left(\mathbf{x}_{i}, \mathbf{x}_{i}^{\prime}\right)_{i=1 \ldots N}$, the relative pointing error between $u$ and $v$ is formally defined by

$$
\frac{1}{N} \sum_{i=1}^{N} \mathrm{~d}\left(\mathbf{x}_{i}^{\prime}, \operatorname{epi}_{u v}^{\mathbf{x}_{i}}(\mathbf{R})\right) .
$$

Here epi $\mathbf{x}_{u v}^{\mathbf{x}_{i}}(\mathbf{R})$ is the epipolar curve of point $\mathbf{x}_{i}$, and $\mathrm{d}$ is the distance, in pixels, between a point and a subset of $\mathbf{R}^{2}$. The set of correspondences between two images can be determined using SIFT (Lowe, 2004). Table 1 gives values for the relative pointing error measured on several Pléiades stereo pairs.

\subsection{Correction of the relative pointing error}

4.2.1 Not absolute but automatic correction The bias affecting the RPC is well known (Fraser and Hanley, 2005). Actually it comes from the sensor attitude estimation, thus also affects the rigorous model, and the RPC approximation is not to blame for it (Fraser and Hanley, 2005). This bias is absolute. It can be evidenced with a single image $u$ and a unique ground control point (GCP) $\mathbf{X}$ by observing that $\mathrm{RPC}_{u}(\mathbf{X})$ is not exactly located on the actual image of $\mathbf{X}$. Several authors have modeled this absolute bias and proposed methods to compensate it (Fraser and Hanley, 2005, Hanley et al., 2002, Grodecki and Dial, 2003). All these methods need GCPs and manual interactions, thus are not suitable in a fully automatic $3 \mathrm{D}$ reconstruction pipeline such as $\mathrm{s} 2 \mathrm{p}$

The relative pointing error can be corrected without any control points. This will not remove the absolute bias affecting the RPC, but will allow to perform efficient stereo matching between the views by following the epipolar curves.

4.2.2 Local relative pointing error Errors within the direct measurement of sensor orientation reside mainly in sensor attitude (Fraser and Hanley, 2005). For an image tile of size $1000 \times 1000$ pixels, covering a scene of size $500 \times 500 \mathrm{~m}$ on the ground (with Pléiades resolution), we can assume that the scene is located at infinity with respect to the satellite. The error can then be modeled in image space as a translation.

A simple way to correct the relative pointing error is thus to transform one of the two images, in such a way that the corresponding points fall on the respective epipolar curves: given two images $u, v$ and a set of correspondences $\left(\mathbf{x}_{i}, \mathbf{x}_{i}^{\prime}\right)_{i=1 \ldots N}$, we search for a translation $\mathrm{T}$ such that, for all $i$, the transformed point $\mathrm{T} \mathbf{x}_{i}^{\prime}$ lies 


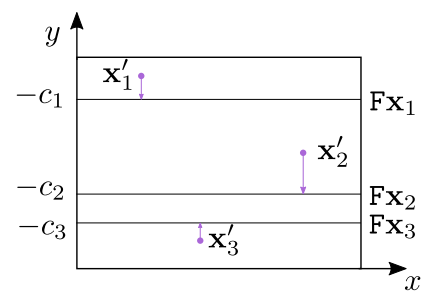

Figure 6: For a tile of size $1000 \times 1000$, the epipolar curves are well approximated by parallel lines (see section 3 ). On this figure the lines are assumed to be horizontal. For each correspondence $\left(\mathbf{x}_{i}, \mathbf{x}_{i}^{\prime}\right)$ there is a vertical shift between the point $\mathbf{x}_{i}^{\prime}$ and the line $\mathbf{F} \mathbf{x}_{i}$. The median of all these shifts minimizes the relative epipolar error defined by formula 2 .

on the epipolar curve epi $\mathrm{i}_{u v}^{\mathbf{x}_{i}}(\mathbf{R})$. The desired translation $\mathrm{T}^{*}$ minimises the relative pointing error defined by equation 2 :

$$
\mathrm{T}^{*}=\underset{\mathrm{T}}{\arg \min } \frac{1}{N} \sum_{i=1}^{N} \mathrm{~d}\left(\mathrm{Tx}_{i}^{\prime}, \mathrm{epi}_{u v}^{\mathbf{x}_{i}}(\mathbf{R})\right) .
$$

From section 3 we know that the epipolar curve epi $\mathbf{x}_{u v}(\mathbf{R})$ is approximated up to 0.05 pixels by the straight line $F \mathbf{x}_{i}$, where $\mathrm{F}$ is the affine fundamental matrix between the two views for the considered tile. As this fundamental matrix is an affine fundamental matrix, all the lines $\mathrm{Fx}_{i}$ are parallel. Without any additional restriction, we may assume that these lines are horizontal (otherwise just do a change of coordinates). The horizontal line $\mathbf{F} \mathbf{x}_{i}$ can be written, in homogeneous coordinates, as

$$
\mathbf{F} \mathbf{x}_{i}=\left[\begin{array}{lll}
0 & 1 & c_{i}
\end{array}\right]^{\top} .
$$

With these notations, for each point correspondence $\left(\mathbf{x}_{i}, \mathbf{x}_{i}^{\prime}\right)$ we have

$$
d\left(\mathbf{x}_{i}^{\prime}, \mathbf{F} \mathbf{x}_{i}\right)=\left|y_{i}^{\prime}+c_{i}\right|,
$$

where $\mathbf{x}_{i}^{\prime}=\left(x_{i}^{\prime}, y_{i}^{\prime}, 1\right)^{\top}$. The situation is illustrated in figure 6. This error is invariant to any horizontal translation, thus the search for a translation minimizing the relative pointing error of formula 3 can be restricted to vertical translations only. With a vertical translation of parameter $t$, the error becomes

$$
\frac{1}{N} \sum_{i=1}^{N} \mathrm{~d}\left(\mathrm{Tx}_{i}^{\prime}, \mathbf{F} \mathbf{x}_{i}\right)=\frac{1}{N} \sum_{i=1}^{N}\left|y_{i}^{\prime}+t+c_{i}\right| .
$$

The translation that minimizes this sum is given by the geometric median (Weiszfeld, 1937) of the vectors $\left(-y_{i}^{\prime}-c_{i}\right)_{i=1 \ldots N}$. The relative pointing error can thus be minimized in a tile by applying a translation to one of the images. Note that the median is robust against outliers, thus this correction procedure works well even in the presence of false matches.

Table 1 gives values of the relative pointing error measured on several Pléiades stereo pairs before and after correction. Figure 7 shows the effect of the corrective translation $\mathrm{T}^{*}$ on the error vectors of a small tile. More details about the proposed procedure can be found in (de Franchis et al., 2014b).

4.2.3 Global relative pointing error model The model we use to correct the pointing error on a tile relies on the validity of the affine approximation. From section 3 we deduce that this model is valid on image regions of size smaller than $1000 \times 1000$ pixels. For bigger regions, the local pointing correction model may not be valid. Several authors (Fraser and Hanley, 2005, Grodecki and Dial, 2003, d'Angelo and Reinartz, 2012) reported that the global RPC bias can be corrected with an affine transformation in image space. A simple way to estimate the optimal

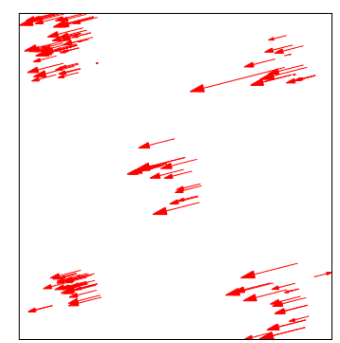

(a)

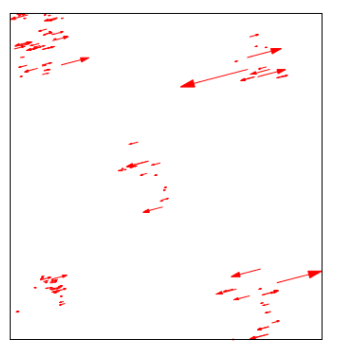

(b)
Figure 7: Error vectors for some keypoints on a $1000 \times 1000$ tile of a Pléiades image. (a) Error vectors before correction. (b) Error vectors after correcting the position of the second image by the optimal translation $\mathrm{T}^{*}$.

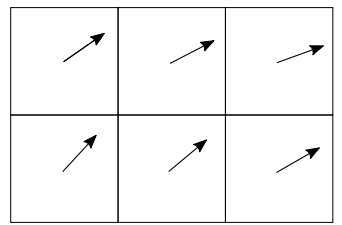

Figure 8: A global pointing correction is estimated for the whole processed region of interest from the local corrections that were computed in each tile. In this example, the region was cut into 6 tiles. In each tile a corrective translation was computed and is represented by an arrow starting from the center of the tile. The 6 corrective translations are used to estimate an affine transformation that corrects the relative pointing error on the whole region.

affine transformation is to use the local corrections computed for each tile. An example of this procedure is given in figure 8 .

\section{STEREO MATCHING AND TRIANGULATION}

For each rectified tile we compute the disparity by applying an off-the-shelf stereo matching algorithm. Because of its performance, we use here the implementation of SGM (Hirschmüller, 2008) included in OpenCV ${ }^{1}$. However, any other stereo matching algorithm can be used instead. The disparities are then interpreted as point correspondences with the coordinates of the original (non rectified) images, as illustrated in figure 9. From these correspondences the $3 \mathrm{D}$ position of the point is triangulated

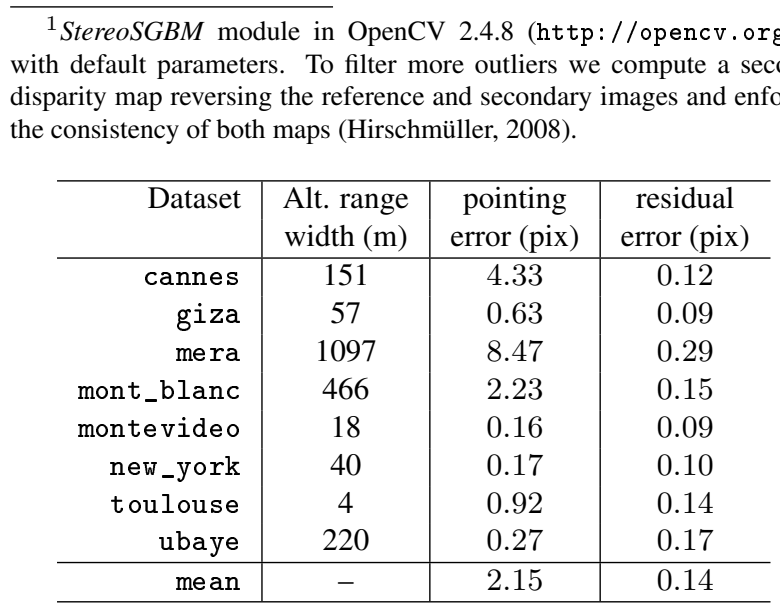

Table 1: Pointing error values before and after correction. On average the correction algorithm reduces the error by a factor 10 . 
using the refined RPC camera models. The SRTM data is used to estimate the initial disparity range, together with the point correspondences that were used to correct the local pointing error.
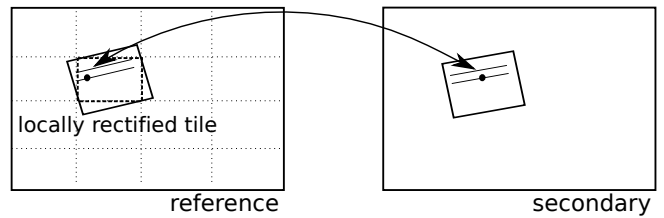

Figure 9: A match on a rectified tile is interpreted as a point correspondence in the coordinate systems of the original images.

Triangulation with RPC functions. As studied in section 4, the limited precision of the RPC data may cause a point $\mathbf{x}^{\prime}$ to be displaced from the epipolar line epi ${ }_{u v}^{\mathbf{x}}(\mathbf{R})$ of the corresponding points $\mathbf{x}$. The Algorithm 2 determines iteratively the altitude of the 3-space point defined by the point correspondence $(\mathbf{x}, \mathbf{q})$, where $\mathbf{q}$ is the projection of $\mathbf{x}^{\prime}$ on the epipolar curve epi $\mathbf{i}_{u v}^{\mathbf{x}}(\mathbf{R})$. The algorithm updates an altitude hypothesis $h$ for the point $\mathbf{x}$ in such a way that its correspondent point $\mathbf{r}_{0}$ is as close as possible to the match $\mathbf{x}^{\prime}$. The altitude hypothesis is updated by linearly approximating the epipolar curve. Figure 10 illustrates the simple rationale behind this algorithm. As the epipolar lines are very smooth the step $h_{S T E P}$ can be large. We set it to 1 .
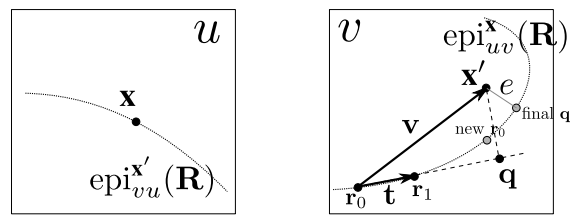

Figure 10: Illustration of one iteration of Algorithm 2. The images $u$ and $v$, two corresponding point $\mathbf{x}$ and $\mathbf{x}^{\prime}$, and the epipolar curve epi $\mathbf{i}_{u v}^{\mathbf{x}}(\mathbf{R})$.

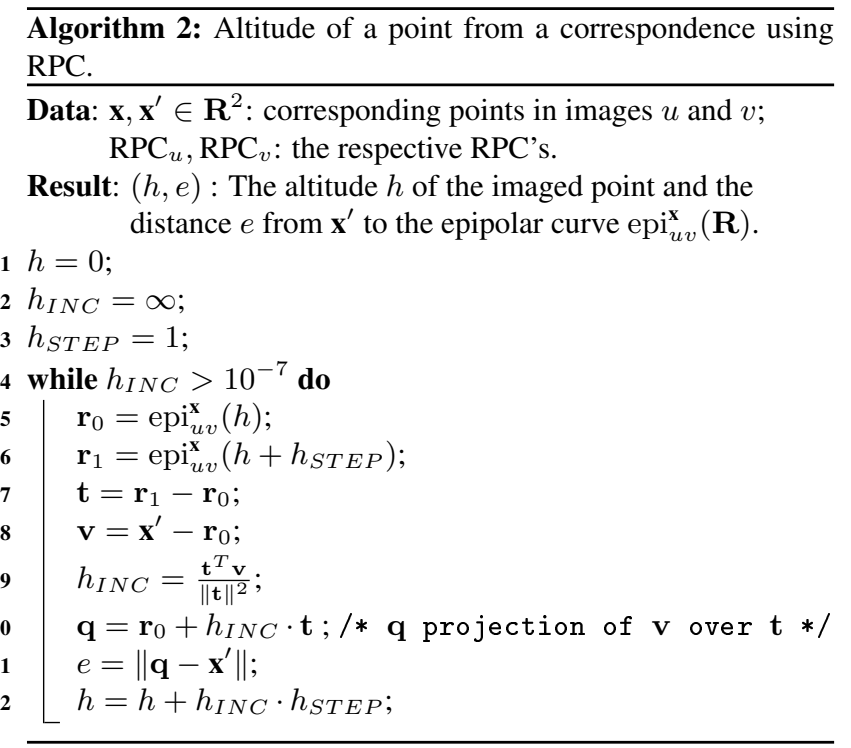

\section{RESULTS AND DISCUSSION}

\subsection{Locally affine rectification}

The stereo image rectification method proposed in section 3 is evaluated by measuring the epipolar error, which is completely determined by the fundamental matrix F. This error is measured

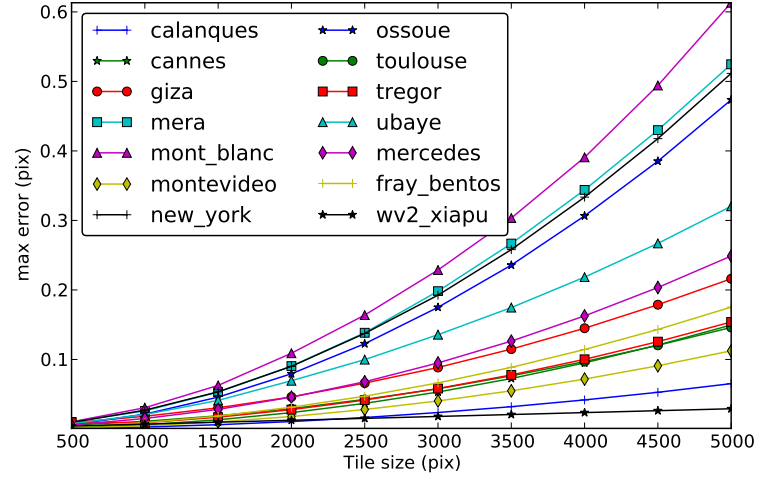

Figure 11: Dependence of the epipolar error with the tile size. A tile of size ranging from $500 \times 500$ to $5000 \times 5000$ pixels was selected in the middle of a Pléiades reference image. Virtual matches were computed using altitude ranges given by SRTM data.

\begin{tabular}{r|c|rl}
\hline Dataset & Scene dim. $(\mathrm{km})$ & \multicolumn{2}{|c}{ RPC altitude validity $(\mathrm{m})$} \\
\hline calanques & $25 \times 24$ & $40-1090$ \\
cannes & $21 \times 20$ & $50-830$ \\
giza & $26 \times 23$ & $10-290$ \\
mera & $25 \times 42$ & $-10-8610$ \\
mont_blanc & $21 \times 15$ & $850-4730$ \\
montevideo & $22 \times 20$ & $-10-150$ \\
new_york & $48 \times 37$ & $-120-190$ \\
ossoue & $22 \times 22$ & $-10-3320$ \\
toulouse & $25 \times 21$ & $150-340$ \\
tregor & $26 \times 24$ & $50-160$ \\
ubaye & $22 \times 15$ & $1100-3050$ \\
mercedes & $25 \times 23$ & $10-90$ \\
fray_bentos & $22 \times 20$ & $0-80$
\end{tabular}

Table 2: Pléiades datasets used for the experiments.

by

$$
\max _{i \in\{1, \ldots, N\}} \max \left\{d\left(\mathbf{x}_{i}^{\prime}, \mathbf{F} \mathbf{x}_{i}\right), d\left(\mathbf{x}_{i}, \mathrm{~F}^{\top} \mathbf{x}_{i}^{\prime}\right)\right\},
$$

where $d(\mathbf{x}, \mathbf{l})$ is the distance, in pixels, between a point $\mathbf{x}$ and a line 1. The matches $\left(\mathbf{x}_{i}, \mathbf{x}_{i}^{\prime}\right)_{i=1 \ldots, N}$ are virtual correspondences obtained as described in section 3.4. This error is the maximal distance between a point's epipolar line and the matching point in the other image (computed for both points of the match). The distance $d\left(\mathbf{x}_{i}^{\prime}, \mathbf{F} \mathbf{x}_{i}\right)$ between a point $\mathbf{x}_{i}^{\prime}$ and the epipolar line it is supposed to lie on, namely $\mathrm{F} \mathbf{x}_{i}$, is computed as

$$
d\left(\mathbf{x}_{i}^{\prime}, \mathbf{F} \mathbf{x}_{i}\right)=\frac{\left|\mathbf{x}_{i}^{\prime \top} \mathrm{F} \mathbf{x}_{i}\right|}{\sqrt{\left(\mathrm{F}_{1}^{\top} \mathbf{x}_{i}\right)^{2}+\left(\mathrm{F}_{2}^{\top} \mathbf{x}_{i}\right)^{2}}}
$$

where $F_{1}^{\top}, F_{2}^{\top}$ and $F_{3}^{\top}$ denote the three rows of matrix $F$.

Numerical results. From a geometric viewpoint, the locally affine rectification method described in section 3 amounts to approximate the two pushbroom sensors with affine camera models. The validity of this approximation relies on the dimensions of the 3 -space domain on which it is used. These dimensions are given by the tile size and the altitude range. To understand the influence of these two parameters, we measured the epipolar error on the Pléiades datasets listed in Table 2.

Figure 11 shows the error measured on each dataset by varying the tile size up to $5000 \times 5000$ pixels, while the altitude range was estimated using SRTM data. These results show that on a 


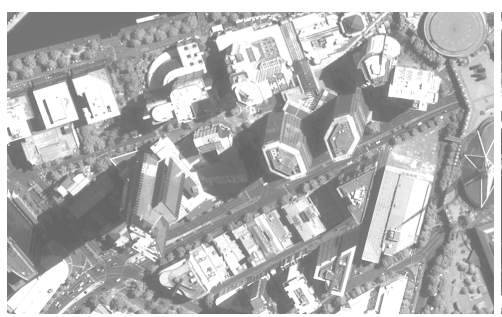

(a) left image

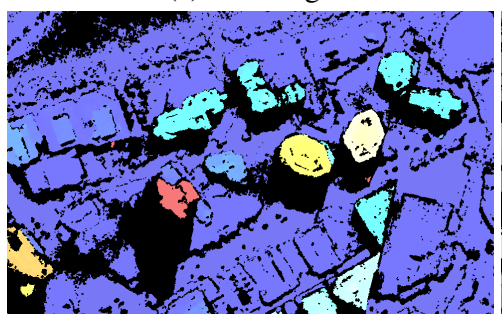

(d) DEM from nadir-left pair

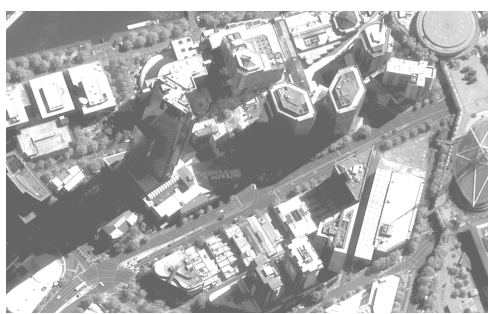

(b) right image

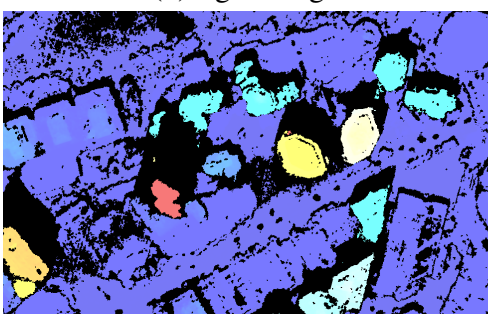

(e) DEM from nadir-right pair

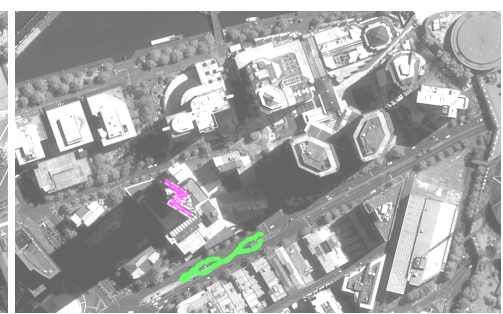

(c) nadir image

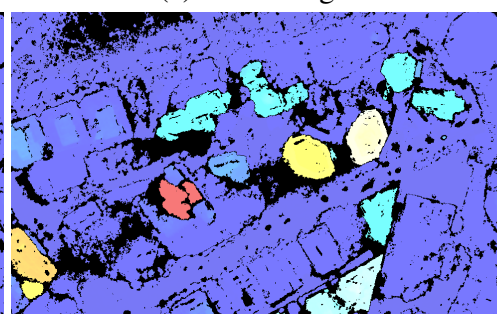

(f) fused DEM

Figure 12: Three Pléiades images of Melbourne (a-c), the roof and street areas used for the evaluation are highlighted in (c). The elevation maps obtained by taking only two images are shown in (d) and (e), while (f) corresponds to the fusion with outlier filtering. Black areas represent rejected pixels.

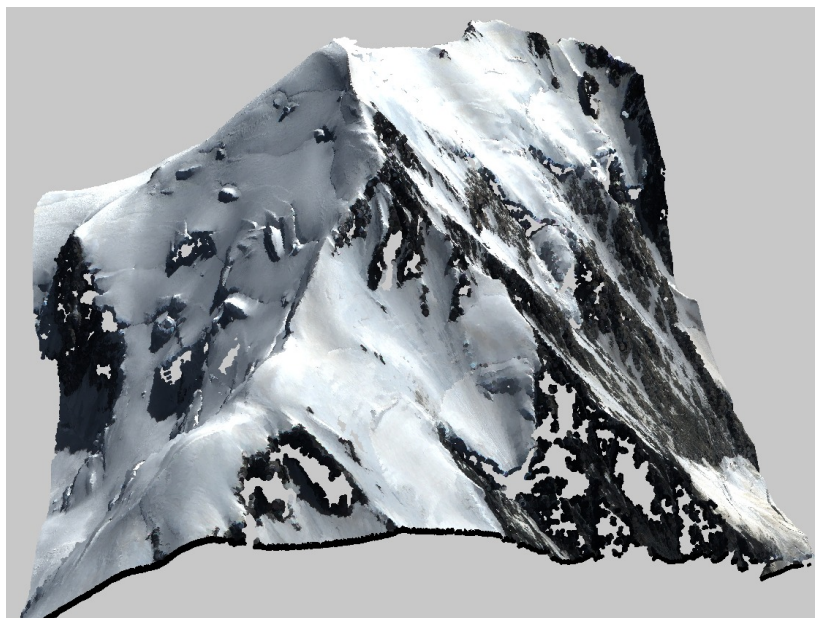

Figure 13: The summit of Mont Blanc, as computed by s2p. To obtain this point cloud, the user clicked a single time on the appropriate place of the map.

Pléiades dataset it is always possible to stereo-rectify tiles of size $1000 \times 1000$ pixels with an epipolar error lower than 0.05 pixels.

\subsection{Pipeline validation}

As a validation of the $\mathrm{s} 2 \mathrm{p}$ pipeline we processed a region from a three-view stereo dataset of Melbourne. Our validation does not include ground control points. Thus we evaluated the relative precision by measuring the height of a known building. The Eureka Tower is a 297.3-metre skyscraper located in the Southbank precinct of Melbourne, which has been highlighted in figure 12(a-c). The altitude estimates were computed by averaging the heights at the street level (yielding 16.15 \pm 0.23 meters) and on the roof (yielding $312.97 \pm 0.49$ meters). Thus our estimated height of the Eureka Tower is $296.82 \pm 0.72$ meters.

Figure 12(d-f) shows the elevation models obtained from the nadir-left and nadir-right pairs for the Melbourne dataset. Note that both images contain significant occluded regions in the vicinity of tall structures, however these regions are complementary.
The fusion of both models exploits this complementarity to produce a much denser elevation model.

As an illustration of the automatic power of s2p, Figure 13 shows the summit of Mont Blanc. This point cloud was obtained after a single click on a Pléiades image. These and other reconstructions can be performed online on the web page associated to this article (de Franchis et al., 2014a).

\section{CODE AND ONLINE DEMO}

The $s 2 p$ stereo pipeline described here is completely implemented and will be released as open source software. It can be tested online (de Franchis et al., 2014a) thanks to the demo framework of the IPOL journal (IPOL, 2010). Several stereo datasets from Pléiades and a stereo pair from WorldView-1 are available for testing. The implementation is compatible with all the stereo datasets provided by Airbus DS and DigitalGlobe, and thus could be tested on images from WorldView-2, QuickBird-1 and Spot-6.

\section{CONCLUSIONS}

Thorough experimentation on numerous Pléiades datasets has shown that using tiles of size $1000 \times 1000$ pixels allows a standard stereo rectification of pushbroom images with a precision of 0.05 pixel, regardless of the altitude range of the scene. The rectification is performed thanks to the RPC data, whose accuracy is locally refined on each tile. This enables standard stereo matching algorithms to be used and tested. An online demo implements the whole $s 2 p$ pipeline for one-click testing.

\section{ACKNOWLEDGEMENTS}

Work partially supported by Centre National d'Etudes Spatiales (MISS Project), European Research Council (Advanced Grant Twelve Labours), Office of Naval Research (under Grant N00014-97-1-0839), Direction Générale de l'Armement, Fondation Mathématique Jacques Hadamard and Agence Nationale de la Recherche (Stereo project). 


\section{REFERENCES}

Astrium, 2012. Pléiades Imagery User Guide version 2.0.

Baltsavias, E. and Stallmann, D., 1992. Metric information extraction from SPOT images and the role of polynomial mapping functions. Int. Arch. Photogramm. Rem. Sens. 29, pp. 358-364.

Christophe, E., Inglada, J. and Giros, A., 2008. Orfeo toolbox: a complete solution for mapping from high resolution satellite images. Int. Arch. Photogramm. Remote Sens. Spatial Inf. Sci 37, pp. 1263-1268.

d'Angelo, P. and Kuschk, G., 2012. Dense multi-view stereo from satellite imagery. In: IGARSS 2012, IEEE, pp. 6944-6947.

de Franchis, C., Facciolo, G. and Meinhardt-Llopis, E., 2014a. s2p online demo. http://dev.ipol.im/ $\operatorname{carlo} / \mathrm{s} 2 \mathrm{p} /$.

de Franchis, C., Meinhardt-Llopis, E., Michel, J., Morel, J.-M. and Facciolo, G., 2014b. Automatic sensor orientation refinement of Pléiades stereo images. In: IGARSS 2014, IEEE.

de Franchis, C., Meinhardt-Llopis, E., Michel, J., Morel, J.-M. and Facciolo, G., 2014c. On stereo rectification of pushbroom images. In: ICIP 2014, IEEE.

d'Angelo, P. and Reinartz, P., 2012. DSM based orientation of large stereo satellite image blocks. Int. Arch. Photogramm. Remote Sens. Spatial Inf. Sci 39(B1), pp. 209-214.

Farr, T. G., Rosen, P. A., Caro, E., Crippen, R., Duren, R., Hensley, S., Kobrick, M., Paller, M., Rodriguez, E., Roth, L. et al., 2007. The shuttle radar topography mission. Rev. Geophys.

Fraser, C. and Hanley, H., 2005. Bias-compensated RPCs for sensor orientation of high-resolution satellite imagery. Photogramm. Eng. Remote Sensing 71(8), pp. 909-915.

Fraser, C., Dare, P. and Yamakawa, T., 2004. Digital surface modelling from SPOT 5 HRS imagery using the affine projective model. In: XXth ISPRS Congress, Vol. XXXV, pp. 385-388.

Grodecki, J. and Dial, G., 2003. Block adjustment of highresolution satellite images described by rational polynomials. Photogramm. Eng. Remote Sensing 69(1), pp. 59-68.

Habib, A. F., Morgan, M., Jeong, S. and Kim, K.-O., 2005. Analysis of Epipolar Geometry in Linear Array Scanner Scenes. The Photogrammetric Record 20(109), pp. 27-47.

Hanley, H., Yamakawa, T. and Fraser, C., 2002. Sensor orientation for high-resolution satellite imagery. Int. Arch. Photogramm. Remote Sens. Spatial Inf. Sci 34(1), pp. 69-75.

Hartley, R. I. and Gupta, R., 1997. Linear Pushbroom Cameras. In: IEEE Trans. Pattern Anal. Mach. Intell., pp. 963-975.

Hartley, R. I. and Zisserman, A., 2004. Multiple View Geometry in Computer Vision. Second edn, Cambridge University Press.

Hirschmüller, H., Scholten, F. and Hirzinger, G., 2005. Stereo Vision Based Reconstruction of Huge Urban Areas from an Airborne Pushbroom Camera (HRSC). In: W. Kropatsch, R. Sablatnig and A. Hanbury (eds), Pattern Recognition, Lecture Notes in Computer Science, Vol. 3663, Springer Berlin, pp. 58-66.

Hirschmüller, H., 2008. Stereo Processing by Semiglobal Matching and Mutual Information. IEEE Trans. Pattern Anal. Mach. Intell. 30(2), pp. 328-341.

IPOL, 2010. Image processing on line. http://www.ipol .im.

Kim, T., 2000. A study on the epipolarity of linear pushbroom images. Photogramm. Eng. Remote Sensing (8), pp. 961-966.

Kuschk, G., 2013. Large scale urban reconstruction from remote sensing imagery. In: 3D-ARCH 2013 - 3D Virtual Reconstruction and Visualization of Complex Architectures, Vol. XL-5/W1, Int. Arch. Photogramm. Remote Sens. Spatial Inf. Sci.

Lee, H.-Y., Kim, T., Park, W. and Lee, H. K., 2003. Extraction of digital elevation models from satellite stereo images through stereo matching based on epipolarity and scene geometry. Image and Vision Computing 21(9), pp. 789-796.

Lowe, D. G., 2004. Distinctive image features from scaleinvariant keypoints. Int. J. Comput. Vis. 60(2), pp. 91-110.
Morgan, M., Kim, K.-O., Jeong, S. and Habib, A., 2006. Epipolar Resampling of Space-borne Linear Array Scanner Scenes Using Parallel Projection. Photogramm. Eng. Remote Sens. 72(11), pp. 1255-1263.

Oh, J., Hee Lee, W., Toth, C. K., Grejner-Brzezinska, D. A. and Lee, C., 2010. A Piecewise Approach to Epipolar Resampling of Pushbroom Satellite Images Based on RPC. Photogramm. Eng. Remote Sens. 76(12), pp. 1353-1363.

Ohta, Y. and Kanade, T., 1985. Stereo by intra- and inter-scanline search using dynamic programming. IEEE Trans. Pattern Anal. Mach. Intell. 7(2), pp. 139-154.

Okamoto, A., Akamatu, S.-I. and Hasegawa, H., 1993. Orientation theory for satellite CCD line-scanner imageries of hilly terrains. Int. Arch. Photogramm. Remote Sens. 29, pp. 217-222.

Ono, T., 1999. Epipolar resampling of high resolution satellite imagery. Int. Arch. Photogramm. Remote Sens.

Orun, A. B. and Natarajan, K., 1994. A modified bundle adjustment software for SPOT imagery and photography: Tradeoff. Photogramm. Eng. Remote Sensing 60(12), pp. 1431-1437.

Tao, C. and Hu, Y., 2001. A comprehensive study of the rational function model for photogrammetric processing. Photogramm. Eng. Remote Sensing 67(12), pp. 1347-1357.

Wang, M., Hu, F. and Li, J., 2011. Epipolar resampling of linear pushbroom satellite imagery by a new epipolarity model. ISPRS J. Photogramm. Remote Sens. 66(3), pp. 347-355.

Weiszfeld, E., 1937. Sur le point pour lequel la somme des distances de $n$ points donnés est minimum. Tôhoku Math. J. 43 , pp. 355-386.

Wohlfeil, J., Hirschmüller, H., Piltz, B., Börner, A. and Suppa, M., 2012. Fully automated generation of accurate digital surface models with sub-meter resolution from satellite imagery. Int. Arch. Photogramm. Rem. Sens. Spatial Inf. Sci 34-B3, pp. 75-80.

\section{A THE RATIONAL POLYNOMIAL CAMERA MODEL}

Each Pléiades image (Astrium, 2012) is accompanied by a pair of functions, called RPC (Baltsavias and Stallmann, 1992, Tao and $\mathrm{Hu}, 2001)$. These functions allow to convert from image coordinates to coordinates on the globe and back. The projection from object space to image plane is denoted by RPC : $\mathbf{R}^{3} \rightarrow \mathbf{R}^{2}$, $(\varphi, \lambda, h) \mapsto(x, y)$, where 3 -space points are represented by their spheroidal coordinates in the World Geodetic System (WGS 84). In that system a point of 3-space is identified by its latitude $\varphi \in[-90,90]$, longitude $\lambda \in]-180,180]$ and altitude $h$, in meters, above the reference ellipsoid. Its inverse, with respect to the first two components, is denoted by $\mathrm{RPC}^{-1}: \mathbf{R}^{3} \rightarrow \mathbf{R}^{3}$, $(x, y, h) \mapsto(\varphi, \lambda, h)$. It takes a point $\mathbf{x}=(x, y)$ in the image domain together with an altitude $h$, and returns the coordinates of the unique 3 -space point $\mathbf{X}=(\varphi, \lambda, h)$ whose altitude is $h$ and whose image is $\mathbf{x}$.

For the sake of clarity, we shall denote by $\mathrm{RPC}_{u}: \mathbf{R}^{3} \rightarrow \mathbf{R}^{2}$ the projection function of the RPC model associated to image $u$, and by $\mathrm{RPC}_{u}^{-1}: \mathbf{R}^{3} \rightarrow \mathbf{R}^{3}$ the corresponding inverse function. Ideally, these functions should verify $\operatorname{RPC}_{u}^{-1}\left(\operatorname{RPC}_{u}(\varphi, \lambda, h), h\right)=$ $(\varphi, \lambda, h)$ and $\mathrm{RPC}_{u}\left(\mathrm{RPC}_{u}^{-1}(x, y, h)\right)=(x, y)$, but as any model the rational polynomial projection has a limited precision. In particular the two RPC functions are not exact inverses of each other. The errors due to concatenating the projection and inverse functions are negligible, being of the order of $10^{-7}$ degrees in WGS 84 coordinates, and $\frac{1}{100}$ of pixel in the image i.e. about $1 \mathrm{~cm}$ on the ground.

Note that Pléiades images are provided by Airbus DS with both $\mathrm{RPC}$ and $\mathrm{RPC}^{-1}$ functions, while DigitalGlobe provides only the projection function RPC. The inverse $\mathrm{RPC}^{-1}$ has to be estimated from RPC. 